\title{
RELAXATION DARK ENERGY IN NON-CRITICAL STRING COSMOLOGIES AND ASTROPHYSICAL DATA
}

\author{
NIKOLAOS E. MAVROMATOS * \\ Physics Department, Theoretical Physics, King's College London, \\ Strand, London WC2R 2LS, UK \\ E-mail: nikolaos.mavromatos@kcl.ac.uk \\ VASILIKI A. MITSOU \\ Instituto de Física Corpuscular (IFIC), CSIC - Universitat de València, \\ Edificio Institutos de Paterna, P.O. Box 22085, E-46071 Valencia, Spain \\ and \\ CERN, Physics Department, CH-1211 Geneva 23, Switzerland \\ E-mail: vasiliki.mitsou@cern.ch
}

\begin{abstract}
In this talk we review briefly the basic features of non-critical (dissipative) String Cosmologies, and we confront some of these models with supernova data. We pay particular attention to the off-shell and dilaton contributions to the dynamical evolution equations of the non-critical string Universe, as well as the Boltzmann equation for species abundances. The latter could have important consequences for the modification of astrophysical constraints on physically appealing particle physics models, such as supersymmetry. The data fits show that non-critical string cosmologies may be viable alternatives to $\Lambda \mathrm{CDM}$ model.

Keywords: Relaxation Dark Energy; Non-Critical String Cosmology; Astrophysical Data.
\end{abstract}

\section{Introduction}

There is a plethora of astrophysical evidence today, from supernovae measurements, ${ }^{1,2}$ the spectrum of fluctuations in the cosmic microwave background, ${ }^{3}$ baryon oscillations ${ }^{4}$ and other cosmological data, indicating that the expansion of the Universe is currently accelerating. The energy budget of the Universe seems to be dominated (by more than 70\%) at the present epoch by a mysterious dark energy component. Many theoretical

${ }^{*}$ Conference Speaker 
models provide possible explanations for the dark energy, ranging from a cosmological constant ${ }^{5}$ to super-horizon perturbations ${ }^{6}$ and time-varying quintessence scenarios, ${ }^{7}$ in which the dark energy is due to a smoothly varying (scalar) field which dominates cosmology in the present era. In the context of string theory, ${ }^{8,9}$ the most successful and mathematically complete theory of quantum gravity available to date, such a time-dependent "quintessence" field is provided by the scalar dilaton field of the gravitational string multiple. ${ }^{10-12}$ The current astrophysical data are capable of placing severe constraints on the nature of the dark energy, whose equation of state may be determined by means of an appropriate global fit. Most of the analyses so far are based on effective four-dimensional RobertsonWalker Universes, which satisfy on-shell dynamical equations of motion of the Einstein-Friedman form. Even in modern approaches to brane cosmology, which are described by equations that deviate during early eras of the Universe from the standard Friedman equation (which is linear in the energy density), the underlying dynamics is assumed to be of classical equilibrium (on-shell) nature, in the sense that it satisfies a set of equations of motion derived from the appropriate minimization of an effective space-time Lagrangian.

However, cosmology may not be an entirely classical equilibrium situation. The initial Big Bang or other catastrophic cosmic event, such as a collision of two brane worlds in the modern approach to strings,,${ }^{9}$ which led to the initial rapid expansion of the Universe, may have caused a significant departure from classical equilibrium dynamics in the early Universe, whose signatures may still be present at later epochs including the present era. One specific model for the cosmological dark energy which is of this type, being associated with a rolling dilaton field that is a remnant of this non-equilibrium phase, was formulated ${ }^{12-14}$ in the framework of non-critical string theory. ${ }^{10,15,16}$ We call this scenario 'Q-cosmology'. It is of outmost importance to confront the currently available precision astrophysical data with such non equilibrium stringy cosmologies. The central purpose of this talk is to present a first step towards this direction, namely a confrontation of cosmological data on high-redshift supernovae ${ }^{17}$ with Q-cosmologies and compare the results with the predictions of the conventional $\Lambda \mathrm{CDM}$ model ${ }^{5}$ and the super-horizon model. ${ }^{6}$ Care must be taken in interpreting the Q-cosmology scenario. Since such a non-equilibrium, non-classical theory is not described by the equations of motion derived by extremising an effective space-time Lagrangian, one must use a more general formalism to make predictions that can be confronted with the current data. 


\section{Dissipative Q-Cosmology Basics}

\subsection{Off-shell (non-critical-string) terms and dissipative cosmological equations}

Q-cosmology are stringy cosmological models, based on the (perturbative) formulation of $\sigma$-models propagating mostly on dilaton and graviton backgrounds, which, however, are not world-sheet conformal invariant. The deviation from conformal invariance may be due to a number of reasons, for instance cosmically catastrophic events at an early stage of the Universe history, such as Big Bang, the collision of two (or more) brane worlds etc. An important formal ingredient of this approach is the identification ${ }^{16}$ of target time with the zero mode $\rho$ of the Liouville field, ${ }^{15}$ which is an extra world-sheet field, introduced in order to restore the conformal invariance of the world-sheet theory. The dynamics of this latter identification is encoded in the solution of the generalized conformal invariance conditions, after Liouville dressing, which read in the $\sigma$-model frame: ${ }^{15,16}$

$$
-\tilde{\beta}^{i}=g^{i^{\prime \prime}}+Q g^{i^{\prime}},
$$

where the prime denotes differentiation with respect to $\rho$, and the overall minus sign on the left-hand side pertains to supercritical strings, ${ }^{10}$ with a timelike signature of the Liouville mode, for which the central charge deficit $Q^{2}>0$ by convention. In physical terms, $Q$ depends on the microscopic details of the specific non-equilibrium string theory model at hand. For instance, in the case of adiabatic brane world collisions with bouncing (recoiling) worlds, ${ }^{12,18,19} Q^{2} \propto v^{4}$, where $v \ll 1$ is the (relative) recoil velocity of the brane worlds after the collision. Notice the dissipation, proportional to the (square root) of the central charge deficit $Q$, on the right-hand side of Eq. (1), which heralds the adjective Dissipative to the associate noncritical-string-inspired Cosmological model. Moreover, the Weyl anomaly coefficients $\tilde{\beta}^{i}, i=\left\{\Phi, g_{\mu \nu}\right\}$, whose vanishing would guarantee local conformal invariance of the string-cosmology background, are associated with off-shell variations of a low-energy effective string-inspired action, $\mathcal{S}\left[g^{j}\right]$ :

$$
\frac{\delta \mathcal{S}[g]}{\delta g^{i}}=\mathcal{G}_{i j} \tilde{\beta}^{j}, \quad \mathcal{G}_{i j}=\operatorname{Lim}_{z \rightarrow 0} z^{2} \bar{z}^{2}\left\langle V_{i}(z, \bar{z}) V_{j}(0,0)\right\rangle,
$$

with $z, \bar{z}$ (complex) world-sheet coordinates, $\mathcal{G}_{i j}$ the Zamolodchikov metric in theory space of strings, ${ }^{8,16}$ and $V_{i}$ the $\sigma$-model vertex operators associated with the $\sigma$-model background field $g^{i}$. It is this off-shell relation that characterises the entire non-critical $(Q)$ Cosmology framework, associated physically with a non-equilibrium situation as a result of an ini- 
tial cosmically catastrophic event, at the beginning of the (irreversible) Liouville/cosmic-time flow.

The detailed dynamics of Eq. (1) are encoded in the solution for the scale factor $a(t)$ and the dilaton $\Phi(t)$ in the simplified model considered in Ref. 14, after the identification of the Liouville mode with the target time. In fact, upon the inclusion of matter backgrounds, the associated equations, after compactification to four target-space dimensions, read in the so-called Einstein frame (i.e., an appropriate redefinition of the $\sigma$-model graviton, such that the graviton-dilaton effective action has a canonically normalised Einstein curvature scalar term $\left.{ }^{10}\right)$ :

$$
\begin{aligned}
& 3 H^{2}-\tilde{\varrho}_{m}-\varrho_{\Phi}=\frac{e^{2 \Phi}}{2} \tilde{\mathcal{G}}_{\Phi}, \quad 2 \dot{H}+\tilde{\varrho}_{m}+\varrho_{\Phi}+\tilde{p}_{m}+p_{\Phi}=\frac{\tilde{\mathcal{G}}_{i i}}{a^{2}}, \\
& \ddot{\Phi}+3 H \dot{\Phi}+\frac{1}{4} \frac{\partial \hat{V}_{\text {all }}}{\partial \Phi}+\frac{1}{2}\left(\tilde{\varrho}_{m}-3 \tilde{p}_{m}\right)=-\frac{3}{2} \frac{\tilde{\mathcal{G}}_{i i}}{a^{2}}-\frac{e^{2 \Phi}}{2} \tilde{\mathcal{G}}_{\Phi}, \\
& \tilde{\mathcal{G}}_{\Phi}=e^{-2 \Phi}\left(\ddot{\Phi}-\dot{\Phi}^{2}+Q e^{\Phi} \dot{\Phi}\right), \\
& \tilde{\mathcal{G}}_{i i}=2 a^{2}\left(\ddot{\Phi}+3 H \dot{\Phi}+\dot{\Phi}^{2}+(1-q) H^{2}+Q e^{\Phi}(\dot{\Phi}+H)\right),
\end{aligned}
$$

where $\tilde{\varrho}_{m}\left(\tilde{p}_{m}\right)$ denotes the matter energy density (pressure), including dark matter contributions, and $\varrho_{\Phi}\left(p_{\Phi}\right)$ are the corresponding quantities for the dilaton dark-energy fluid. The overdots in the above equations denote derivatives with respect to the Einstein time, which is related to the Robertson-Walker cosmic time $t_{R W}$ by $t=\omega t_{R W}$. Without loss of generality we have taken $\omega=\sqrt{3} H_{0}$ where $H_{0}$ is the present-day Hubble parameter. With this choice for $\omega$ the densities appearing in Eqs. (3) are given in units of the critical density. In the above equations, $H=\dot{a} / a$ is the Hubble parameter and $q$ is the deceleration parameter of the Universe $q \equiv-\ddot{a} a / \dot{a}^{2}$, and are both functions of (Einstein frame) cosmic time. The potential $\hat{V}_{\text {all }}$ appearing above is defined by $\hat{V}_{\text {all }}=2 Q^{2} \exp (2 \Phi)+V$ where, in order to cover more general cases, we have also allowed for a (e.g., string-loop-induced) potential term in the four-dimensional action, $-\int d^{4} y \sqrt{-G} V$. We have also assumed a (spatially) flat Universe. The dilaton energy density and pressure are given in this class of models by: $\varrho_{\Phi}=\frac{1}{2}\left(2 \dot{\Phi}^{2}+\hat{V}_{\text {all }}\right), p_{\Phi}=\frac{1}{2}\left(2 \dot{\Phi}^{2}-\hat{V}_{\text {all }}\right)$. The dependence of the central charge deficit $Q(t)$ on the cosmic time stems from the running of the latter with the world-sheet RG scale, ${ }^{16,19}$ and is provided by the Curci - Paffuti equation ${ }^{20}$ expressing the renormalisability of the world-sheet theory. To leading order in an $\alpha^{\prime}$ expansion, which we restrict ourselves here, this equation in the Einstein frame reads: ${ }^{14} \frac{d \tilde{\mathcal{G}}_{\Phi}}{d t_{E}}=-6 e^{-2 \Phi}(H+\dot{\Phi}) \frac{\tilde{\mathcal{G}}_{i i}}{a^{2}}$. For completeness we also state here the continuity equation of the matter stress tensor, which is not an indepen- 
dent equation, but can be obtained from Eq. (3) by appropriate algebraic manipulations: $\frac{d \tilde{\varrho}_{m}}{d t_{E}}+3 H\left(\tilde{\varrho}_{m}+\tilde{p}_{m}\right)+\frac{\dot{Q}}{2} \frac{\partial \hat{V}_{a l l}}{\partial Q}-\dot{\Phi}\left(\tilde{\varrho}_{m}-3 \tilde{p}_{m}\right)=6(H+\dot{\Phi}) \frac{\tilde{\mathcal{G}}_{i i}}{a^{2}}$.

A consistent numerical solution of $a(t), \Phi(t)$, and the various densities, including back reaction of matter onto the space-time geometry, has been discussed in Ref. 14, where we refer the interested reader for further study. We note the existence of exotic matter scaling in this approach today, which no longer scales as dust, but it includes different scaling components, e.g. $a^{-\delta}$, with $\delta$ close to 4 . We quote below the final result of our parametrisation for $H(z)$ in the Q-cosmology framework, at late eras, such as the ones pertinent to the supernova and other data $(0<z<2)$, where some analytic approximations are allowed: ${ }^{17}$

$$
\frac{H(z)}{H_{0}}=\sqrt{\Omega_{3}(1+z)^{3}+\Omega_{\delta}(1+z)^{\delta}+\Omega_{2}(1+z)^{2}}, \quad \Omega_{3}+\Omega_{\delta}+\Omega_{2}=1,
$$

with the densities $\Omega_{2,3, \delta}$ corresponding to present-day values $(z=0)$. We stress, however, that a complete analysis of the non-critical and dilaton effects, which turn out to be important in the present era after the inclusion of matter, requires numerical treatment. ${ }^{14}$ In view of this, the exponent $\delta$ is treated from now on as a fitting parameter, and in fact it may even be $z$-dependent to cover more general cases, and get agreement with the numerical treatment of Ref. 14. It is important to note that the various $\Omega_{i}$ contain contributions from both dark energy and matter energy densities. As explained in detail in Ref. $17, \Omega_{3}$ does not merely represent ordinary matter effects, but also receives contributions from the dilaton dark energy. In fact, the sign of $\Omega_{3}$ depends on details of the underlying theory, and it could even be negative. For instance, Kaluza-Klein graviton modes in certain brane-inspired models ${ }^{21}$ yield negative dust contributions. In a similar vein, the exotic contributions scaling as $(1+z)^{\delta}$ are affected by the off-shell Liouville terms of Q-cosmology. It is because of the similar scaling behaviours of dark matter and dilaton dark energy that we reverted to the notation $\Omega_{i}, i=2,3, \delta$, in Eq. (4). To disentangle the ordinary matter and dilaton contributions one may have to resort to further studies on the equation of state of the various components, which we do not study here or in. ${ }^{17}$ More generally, one could have included a cosmological constant $\Omega_{\Lambda}$ contribution in Eq. (4), which may be induced in certain brane-world inspired models. We do not do so in this work, as our primary interest is to fit Q-cosmology models, ${ }^{12-14}$ which are characterised by dark energy densities that relax to zero. 


\subsection{Dilaton and off-shell modifications to cosmic evolution of species abundances}

The above formalism can be used to derive the dilaton and non-criticalstring effects on Boltzmann-type evolution equations ${ }^{22}$ of a number of densities for certain particle physics species, playing the rôle of dominant dark matter candidates. In the presence of (time-dependent) dilaton and off-shell graviton fields, that couple to matter, the phase-space density of species depends on sources, $f\left(|\vec{p}|, t, \Phi(t, \rho), g_{\mu \nu}(t, \rho)\right)$, where $p$ is the momentum, and $\rho$ is the Liouville mode, which eventually is going to be identified with a function of the target time $t,{ }^{16}$ whose form is dictated by both conformalfield-theory and target-space dynamical considerations, such as the minimisation of the effective potential. ${ }^{18} \mathrm{~A}$ detailed analysis ${ }^{23}$ shows that the Boltzmann equation for the density of species $X$, with mass $m_{X}$, assumed to be a dominant dark matter candidate, $n=\int d^{3} p f$, can be written in a compact form that represents collectively the dilaton-dissipative-source and non-critical-string contributions as external-source $\Gamma(t) n$ terms:

$$
\frac{d n}{d t}+3 \frac{\dot{a}}{a} n=\Gamma(t) n-\langle v \sigma\rangle\left(n^{2}-n_{e q}^{2}\right), \quad \Gamma(t) \equiv \dot{\Phi}+\frac{1}{2} \eta e^{-\Phi} g^{\mu \nu} \tilde{\beta}_{\mu \nu}^{\mathrm{Grav}}
$$

where the last term on the right-hand-side of the Boltzman equation is the collision term, expressed in terms of the thermal average of the cross section $\sigma$ times the Möller velocity $v$ of the annihilated particles; ${ }^{22} \eta$ is a worldsheet renormalization-scheme dependent parameter; ${ }^{16,23}$ for our purposes here we work in the physical scheme $\eta=-1$. To find an explicit expression for $\Gamma(t)$ in our case requires the full solution of the Q-cosmological equations. ${ }^{14}$ Depending on the sign of $\Gamma(t)$ one has different effects on the relic abundance of the species $X$ with density $n$, which we now proceed to analyse briefly. Before the decoupling ('freeze out') time $t_{f}, t<t_{f}$, equilibrium is maintained and thus $n=n_{e q}$ for such an era. However, it is crucial to observe that, as a result of the presence of the source $\Gamma$ terms, $n_{e q}$ no longer scales with the inverse of the cubic power of the expansion radius $a$, which was the case in conventional (on-shell) cosmological models. Under some plausible assumptions, for instance that the entropy remains approximately constant after the freeze out temperature, and that one can define a temperature $T$ for the ordinary matter degrees of freedom, but not for the ones pertaining to (dilatonic) dark energy, we can solve the Boltzmann equation and arrive at the dilaton and non-critical string corrections to the freeze out temperature of, say, the lightest supersymmetric particle, $\tilde{\chi}$, assumed 
to be the dominant dark matter candidate (we define $x \equiv T / m_{\tilde{\chi}}$ ):

$$
x_{f}^{-1}=\ln \left[\left(0.03824 g_{s} \frac{M_{\text {Planck }} m_{\tilde{\chi}}}{\sqrt{g_{*}}} x_{f}^{1 / 2}\langle v \sigma\rangle_{f}\right)\left(\frac{g_{*}}{\tilde{g}_{*}}\right)^{1 / 2}\right]+\int_{x_{f}}^{x_{i n}} \frac{\Gamma d x}{H x},(6)
$$

whereby $\tilde{g}_{\text {eff }}$ is simply defined by ${ }^{23} \varrho+\Delta \varrho \equiv \frac{\pi^{2}}{30} T^{4} \tilde{g}_{\text {eff }}$. $\Delta \varrho$ incorporates the effects of the additional contributions due to the non-critical (off-shell) terms and the dilaton dissipative source, which are not accounted for by the ordinary matter degrees of freedom $g_{\text {eff }}$ of conventional Cosmology. ${ }^{22}$ Recalling that only the degrees of freedom involved in ordinary matter energy density $\rho$ are thermal, ${ }^{22} \rho=\frac{\pi^{2}}{30} T^{4} g_{\text {eff }}(T)$, whilst from the dynamical equations of Q-cosmology $H^{2}=\frac{8 \pi G_{N}}{3}(\rho+\Delta \rho)$, we obtain ${ }^{23} \tilde{g}_{\text {eff }}=g_{\text {eff }}+$ $\frac{30}{\pi^{2}} T^{-4} \Delta \rho$. We can also assume that the $\tilde{\chi}$ 's decoupled before neutrinos. Then, for the relic abundance, we derive the following approximate result ${ }^{23}$

$$
\Omega_{\tilde{\chi}} h_{0}^{2}=\left(\Omega_{\tilde{\chi}} h_{0}^{2}\right)_{n o-\text { source }} \times\left(\frac{\tilde{g}_{*}}{g_{*}}\right)^{1 / 2} \exp \left(\int_{x_{0}}^{x_{f}} \frac{\Gamma d x}{H x}\right),
$$

with $\left(\Omega_{\tilde{\chi}} h_{0}^{2}\right)_{n o-\text { source }}=\frac{1.066 \times 10^{9} \mathrm{GeV}^{-1}}{M_{\text {Planck } \sqrt{g_{*}} J}}, \quad J \equiv \int_{x_{0}}^{x_{f}}\langle v \sigma\rangle d x$, and $x_{f}$ determined by Eq. (6). The merit of casting the relic density in such a form is that it clearly exhibits the effect of the presence of the source. It is immediately seen from Eq. (7) that, depending on the sign of the source $\Gamma$, one may have increase or reduction of the relic density as compared with the corresponding value in the absence of the source. In this way, the constraints imposed on supersymmetric extensions of the Standard Model from astrophysical data, ${ }^{24}$ such as those from WMAP-satellite measurements, ${ }^{3}$ etc., based on the available bounds of, say, cold dark matter relic densities, need to be revisited. This is left for the future.

\section{Confronting Q-Cosmologies with Supernova Data}

We use the type-Ia supernovae (SN) data reported in Refs. 1,2, which are given in terms of the distance modulus $\mu=5 \log d_{L}+25$, where the luminosity distance $d_{L}$ (in megaparsecs) is related to the redshift $z$ via the Hubble rate $H$ :

$$
d_{L}=c(1+z) \int_{0}^{z} \frac{d z^{\prime}}{H\left(z^{\prime}\right)} .
$$

We note that this observable depends on the expansion history of the Universe from $z$ to the present epoch, and recall that most of the available supernovae have $z<1$, although there is a handful with larger values of $z$. 
In the analysis that follows, the predictions of the following three cosmological models are investigated:

$\Lambda$ CDM model assuming a flat universe, where the Hubble rate is

$$
H(z)=H_{0}\left(\Omega_{\mathrm{M}}(1+z)^{3}+\Omega_{\Lambda}(1+z)^{3\left(1+w_{0}\right)}\right)^{1 / 2} .
$$

Super-horizon model in which the Hubble parameter is given by ${ }^{6}$

$$
H(z)=\bar{a}^{-1} \frac{d \bar{a}(t)}{d t}=\frac{\bar{H}_{0}}{1-\Psi_{\ell 0}}\left(a^{-3 / 2}-a^{-1 / 2} \Psi_{\ell 0}\right),
$$

where $(1+z)^{-1}=\bar{a}(t)$ and $\Psi_{\ell 0}$ is a free parameter.

Q-cosmology discussed in the previous sections, with $H$ given by Eq. (4) and three parameters to be determined by the fit: $\Omega_{3}, \Omega_{\delta}$ and $\delta$.

Measurements are available of the distance moduli of 157 supernovae in a so-called 'gold' sample, ${ }^{1}$ observed by ground-based facilities and the Hubble Space Telescope (HST). In addition, a so-called 'silver' dataset of 29 SN 1a is also available, of a slightly lesser spectrometric and photometric quality. The analysis was also repeated including the 'silver' supernovae data with a total of $186 \mathrm{SN}$ - with results comparable to those with the 'gold' dataset, proving the robustness of the analysis. Furthermore, we analysed the measurements of 71 other high-redshift supernovae by the Supernova Legacy Survey (SNLS), ${ }^{2}$ which are accompanied by a reference sample of 44 nearby SN Ia, yielding a total of 115 data points. Since the fits to the two datasets are quite compatible, ${ }^{17}$ a combined analysis was finally performed.

For illustration purposes, both data and predictions of cosmological models are expressed in the following as residuals, $\Delta \mu$, from the emptyUniverse $\left(\Omega_{\mathrm{M}}=0\right)$ prediction. The combined sample of the 'gold' (157 SN) plus the SNLS sample (71 SN), yielding a sample of 228 supernovae in total, is shown in Fig. 1, where the predictions of the cosmological models under study are also displayed for the best-fit parameter values.

The analysis involves minimisation of the standard $\chi^{2}$ function with respect to the cosmological model parameters. The best-fit parameter values acquired by combining the 'gold' sample and the SNLS supernovae datasets, the $1 \sigma$ errors and the corresponding $\chi^{2}$ values are listed in Table 1 for the three cosmological models.

It is evident from Fig. 1 and Table 1 that the standard $\Lambda$ CDM model fits the supernova data very well, as expected from earlier analyses. The super-horizon dark matter model also fits the supernova data quite well. Both of these models are on-shell, i.e., they satisfy the pertinent Einstein's 


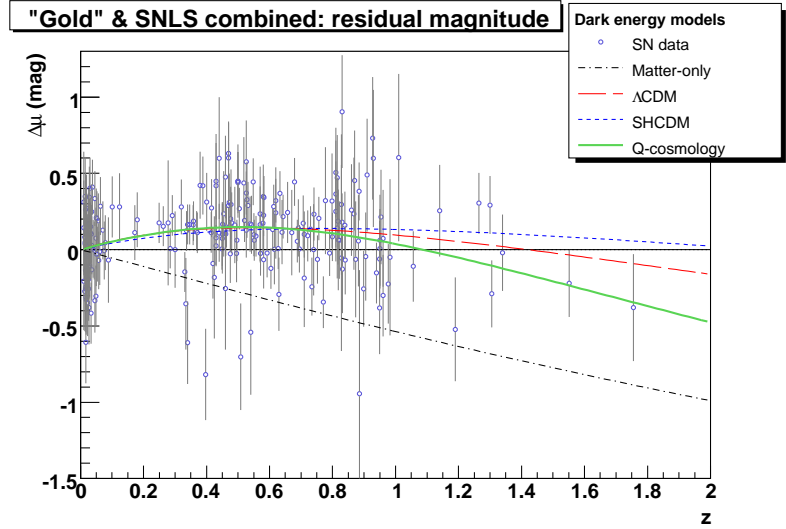

Fig. 1. Residual magnitude versus redshift for supernovae from the 'gold' and the SNLS datasets. Predictions of cosmological models for the best-fit parameter values are superimposed: (i) Empty Universe; (ii) Universe with matter only, $\Omega_{\mathrm{M}}=1$; (iii) $\Lambda$ CDM model; (iv) super-horizon model; and (v) off-shell Q-cosmology model.

Table 1. Parameter values favoured by the 'gold' + SNLS combined data for various cosmological models.

\begin{tabular}{lccc}
\hline Models & Best-fit parameters & $\chi^{2}$ & $\chi^{2} /$ dof \\
\hline$\Lambda$ CDM & $\Omega_{\mathrm{M}}=0.274 \pm 0.017$ & 239 & 1.05 \\
Super-horizon & $\Psi_{\ell 0}=-0.87 \pm 0.06$ & 245 & 1.09 \\
& $\Omega_{3}=-3.7 \pm 1.1$ & & \\
Q-cosmology & $\Omega_{\delta}=1.3 \pm 0.7$ & 237 & 1.05 \\
& $\delta=3.9 \pm 0.3$ & & \\
\hline
\end{tabular}

equations. Moreover, off-shell cosmology models are also compatible with the data. As we discussed above, off-shell effects are important in our Qcosmology model. Introducing the appropriate parametrisation (4) to allow for these off-shell effects, we find that the Q-cosmology model may fit the supernovae data as well as the standard $\Lambda C D M$ model.

In Fig. 2, the Hubble parameter predictions of the various models are compared with the data available from high- $z$ red galaxies ${ }^{25,26}$ and from sets of type Ia SN. ${ }^{27}$ The bands correspond to the $68 \%$ confidence intervals deduced by the aforementioned analysis of 228 supernovae (cf. Table 1). The SN data constrain $H$ only up to $z \simeq 1.3$ with a $50 \%$ uncertainty, in contrast to the red galaxies data, which provide stringent measurement of $H$ until $z \simeq 1.8$. The $\Lambda$ CDM model is compatible with the data, however the super-horizon model deviates from both galaxies and supernovae data. 
Q-cosmology, on the other hand, predict higher values of $H$ than the galaxies data measurements, though fully compatible with the SN data, as expected. Nevertheless, this discrepancy is hoped to be remedied by repeating the analysis of Ref. 17 with the inclusion of a sample of 21 type-Ia supernovae -including 13 with $z>1$ - recently released by HST. ${ }^{27}$ Moreover, as remarked earlier, a $z$-dependent- $\delta$ parametrisation of $H(z)(4)$ may be necessary, as becomes clear from the highly non-linear form of the numerical solution of some Q-cosmology models presented in Ref. 14. In fact, fitting the numerical solution itself with the data is probably the most complete treatment. We hope to tackle such issues in the future.



Fig. 2. The Hubble parameter redshift relation for various cosmological models and observational data. The bands represent $68 \%$ confidence intervals derived by the SN analysis of Ref. 17 for the standard $\Lambda$ CDM, the super-horizon and the Q-cosmology models. The black rectangle shows the WMAP3 ${ }^{3}$ estimate for $H_{0}$, the squares show the measurements from SDSS galaxies, ${ }^{25}$ the triangles result from high- $z$ red galaxies,${ }^{26}$ and the circles correspond to a combined analysis ${ }^{27}$ of supernovae data from Refs. 1 and 2.

For standard on-shell cosmologies, complementary information is provided by the data on baryon acoustic oscillations, ${ }^{4}$ which show up in the galaxy-galaxy correlation function at $z \sim 0.35$. However, for reasons discussed in Ref. 17, the application of such an analysis to the off-shell Qcosmology model is an open issue, since the underlying theoretical framework needs to be re-evaluated.

\section{Conclusions}

In this talk, we briefly reviewed the main predictions of Q-cosmologies, including a brief discussion about the time-dependent-dilaton and noncritical-string effects on the Boltzmann equation for species abundances, ${ }^{23}$ 
which could lead to important modifications of the constraints imposed by astro-physical data on interesting particle physics models, such as supersymmetric extensions of the standard model. Then, we discussed some initial steps towards demonstrating ${ }^{17}$ that the available supernova data are compatible with such non-critical-string-based cosmologies, ${ }^{12,14}$ thus implying the possibility that such models may be viable alternatives to the Standard $\Lambda$ CDM model. As more precision astrophysical data are coming into play, more stringent constraints can be imposed on our non-critical string Q-cosmologies.

Acknowledgements: We thank G. Diamandis, J. Ellis, B. Georgalas, A. Lahanas and D. Nanopoulos for the enjoyable collaboration and discussions. The work of N.E.M. was supported in part by the European Union through the Marie Curie Research and Training Network UniverseNet (MRTN-CT-2006-035863).

\section{References}

1. A. G. Riess et al. [Supernova Search Team Collaboration], Astrophys. J. 607, 665 (2004) [arXiv:astro-ph/0402512].

2. P. Astier et al., Astron. Astrophys. 447 (2006) 31 [arXiv:astro-ph/0510447].

3. D. N. Spergel et al., arXiv:astro-ph/0603449.

4. D. J. Eisenstein et al., Astrophys. J. 633 (2005) 560 [arXiv:astroph/0501171]; S. Cole et al. [The 2dFGRS Collaboration], Mon. Not. Roy. Astron. Soc. 362, 505 (2005) [arXiv:astro-ph/0501174].

5. N. A. Bahcall, J. P. Ostriker, S. Perlmutter and P. J. Steinhardt, Science 284, 1481 (1999) [arXiv:astro-ph/9906463].

6. E. W. Kolb, S. Matarrese, A. Notari and A. Riotto, arXiv:hep-th/0503117; E. W. Kolb, S. Matarrese and A. Riotto, arXiv:astro-ph/0506534.

7. A. Upadhye, M. Ishak and P. J. Steinhardt, Phys. Rev. D 72 (2005) 063501 [arXiv:astro-ph/0411803].

8. M.B. Green, J.H. Schwarz and E. Witten, Superstring Theory, Vols. I \& II (Cambridge University Press, 1987).

9. J. Polchinski, String theory, Vols. I \& II (Cambridge University Press, 1998).

10. I. Antoniadis, C. Bachas, J. R. Ellis and D. V. Nanopoulos, Phys. Lett. B 211, 393 (1988); Nucl. Phys. B 328, 117 (1989); Phys. Lett. B 257, 278 (1991).

11. M. Gasperini, F. Piazza and G. Veneziano, Phys. Rev. D 65, 023508 (2002) [arXiv:gr-qc/0108016].

12. J. R. Ellis, N. E. Mavromatos and D. V. Nanopoulos, Phys. Lett. B 619, 17 (2005) [arXiv:hep-th/0412240]; J. R. Ellis, N. E. Mavromatos, D. V. Nanopoulos and M. Westmuckett, Int. J. Mod. Phys. A 21 (2006) 1379 [arXiv:gr-qc/0508105].

13. G. A. Diamandis, B. C. Georgalas, N. E. Mavromatos and E. Papantonopou- 
los, Int. J. Mod. Phys. A 17, 4567 (2002) [arXiv:hep-th/0203241]; G. A. Diamandis, B. C. Georgalas, N. E. Mavromatos, E. Papantonopoulos and I. Pappa, Int. J. Mod. Phys. A 17, 2241 (2002) [arXiv:hep-th/0107124].

14. G. A. Diamandis, B. C. Georgalas, A. B. Lahanas, N. E. Mavromatos and D. V. Nanopoulos, Phys. Lett. B 642, 179 (2006) [arXiv:hep-th/0605181].

15. F. David, Mod. Phys. Lett. A 3, 1651 (1988); J. Distler and H. Kawai, Nucl. Phys. B 321, 509 (1989); J. Distler, Z. Hlousek and H. Kawai, Int. J. Mod. Phys. A 5, 391 (1990); see also: N. E. Mavromatos and J. L. Miramontes, Mod. Phys. Lett. A 4, 1847 (1989); E. D'Hoker and P. S. Kurzepa, Mod. Phys. Lett. A 5, 1411 (1990).

16. J. R. Ellis, N. E. Mavromatos and D. V. Nanopoulos, Phys. Lett. B 293, 37 (1992) [arXiv:hep-th/9207103]; Mod. Phys. Lett. A 10, 1685 (1995) [arXiv:hep-th/9503162]. Invited review for the special Issue of J. Chaos Solitons Fractals, Vol. 10, (eds. C. Castro amd M.S. El Naschie, Elsevier Science, Pergamon 1999) 345 [arXiv:hep-th/9805120];

17. J. R. Ellis, N. E. Mavromatos, V. A. Mitsou and D. V. Nanopoulos, arXiv:astro-ph/0604272, Astroparticle Physics in press.

18. E. Gravanis and N. E. Mavromatos, Phys. Lett. B 547, 117 (2002) [arXiv:hepth/0205298]; N. E. Mavromatos, arXiv:hep-ph/0309221.

19. J. R. Ellis, N. E. Mavromatos, D. V. Nanopoulos and A. Sakharov, New J. Phys. 6, 171 (2004) [arXiv:gr-qc/0407089];

20. G. Curci and G. Paffuti, Nucl. Phys. B 286, 399 (1987).

21. M. Minamitsuji, M. Sasaki and D. Langlois, Phys. Rev. D 71, 084019 (2005) [arXiv:gr-qc/0501086].

22. E. W. Kolb and M. S. Turner, The Early Universe, Front. Phys. 69 (Redwood City, USA: Addison-Wesley 1990).

23. A. B. Lahanas, N. E. Mavromatos and D. V. Nanopoulos, arXiv:hep$\mathrm{ph} / 0608153$.

24. A. B. Lahanas, D. V. Nanopoulos and V. C. Spanos, Phys. Lett. B 518, 94 (2001) [arXiv:hep-ph/0107151]; J. R. Ellis, K. A. Olive, Y. Santoso and V. C. Spanos, Phys. Lett. B 565, 176 (2003) [arXiv:hep-ph/0303043]; U. Chattopadhyay, A. Corsetti and P. Nath, Phys. Rev. D 68, 035005 (2003) [arXiv:hep-ph/0303201]; for a review see: A. B. Lahanas, N. E. Mavromatos and D. V. Nanopoulos, Int. J. Mod. Phys. D 12, 1529 (2003) [arXiv:hep$\mathrm{ph} / 0308251]$ and references therein.

25. R. Jimenez, L. Verde, T. Treu and D. Stern, Astrophys. J. 593 (2003) 622 [arXiv:astro-ph/0302560].

26. J. Simon, L. Verde and R. Jimenez, Phys. Rev. D 71 (2005) 123001 [arXiv:astro-ph/0412269].

27. A. G. Riess et al., arXiv:astro-ph/0611572. 